\title{
Comparison of circular stapling, triangulating stapling and T-shape stapling for cervical anastomosis with minimally invasive esophagectomy
}

\author{
Yu $\operatorname{Tian}^{1}, \operatorname{Lin} \mathrm{Li}^{2}$, Shuhai $\mathrm{Li}^{2}$, Hui Tian ${ }^{2}, \operatorname{Ming} \mathrm{Lu}^{2,3}$ \\ ${ }^{1}$ Department of Thoracic Surgery, The Second Hospital of Shandong University, Jinan, China; ${ }^{2}$ Department of Thoracic Surgery, Qilu Hospital, \\ Shandong University, Jinan, China; ${ }^{3}$ School of Medicine, Shandong University, Jinan, China \\ Contributions: (I) Conception and design: M Lu, H Tian; (II) Administrative support: H Tian; (III) Provision of study materials or patients: L Li; (IV) \\ Collection and assembly of data: Y Tian; (V) Data analysis and interpretation: Y Tian, S Li; (VI) Manuscript writing: All authors; (VII) Final approval \\ of manuscript: All authors. \\ Correspondence to: Ming Lu, PhD. Department of Thoracic Surgery, Qilu Hospital, Shandong University, 107\# Wenhua Xi Road, Jinan 250012, \\ China. Email: lumingqilu@126.com.
}

\begin{abstract}
Background: The triangulating stapling (TST) and T-shape stapling (TS) methods have been proposed to decrease the incidence of anastomotic leakage after esophagectomy, but few studies have compared them to the circular stapling technique (CS). This retrospective study aimed to compare the efficacy and safety of three cervical anastomosis methods after esophagectomy.

Methods: Squamous cell carcinoma patients who underwent minimally invasive esophagectomy (MIE) with CS, TST or TS anastomosis between April 2010 and June 2012 were recruited. Their clinical characteristics and short-term outcome were analyzed. Kaplan-Meier analyses compared with log-rank test were used to calculate the effect of the three types of cervical anastomosis on overall survival (OS) and disease-free survival (DFS).

Results: The incidence of anastomotic leakage was $21.8 \%$ in the CS group, $7.7 \%$ in the TS group and $11.9 \%$ in the TST group ( $\mathrm{P}=0.029)$. There were significant differences in the incidence of gastroesophageal reflux among the three groups $(\mathrm{P}<0.001)$. Rates of anastomotic stenosis, pulmonary infection, chylothorax and hoarseness were not different among the groups. There were significant differences in anastomotic time, operation time and hospitalization time (all $\mathrm{P}<0.001$ ), but there was no significant difference in albumin content at 1 month after operation $(\mathrm{P}=0.226)$. There was no differences in long-term surgical effects of the three types of anastomosis
\end{abstract}

Conclusions: Cervical esophagogastric anastomosis by TST or TS can be considered feasible and safe and with improved short-term outcome.

Keywords: Anastomotic leakage; anastomotic techniques; T-shape stapling (TS); triangulating stapling (TST)

Submitted Oct 09, 2020. Accepted for publication Dec 02, 2020.

doi: $10.21037 /$ atm-20-7278

View this article at: http://dx.doi.org/10.21037/atm-20-7278

\section{Introduction}

Surgical resection is an important and effective treatment for esophageal cancer. Anastomotic techniques are key to digestive tract reconstruction after esophagectomy (1), but there are many complications, such as anastomotic leakage, anastomotic stenosis and anastomotic reflux (1-5).
Currently, most neck anastomoses use the circular stapling technique (CS) for tubular anastomoses, because it is simple and has a low rate of leakage. However, if there is anastomotic leakage after surgery, the stricture of the anastomosis in the neck results in a poor outcome and longterm decline in quality of life after surgery $(2,6-8)$. 
The triangulating stapling (TST) and T-shape stapling (TS) methods of anastomosis use a linear stapler. The rate of anastomotic leakage is higher with a TST anastomosis than with a CS tubular anastomosis $(9,10)$, but TS anastomosis has shown good efficacy and safety, and is commonly used in thoracic laparoscopic Ivor Lewis esophagectomy anastomosis; however, the length of the residual esophagus in the neck limits the use of TS for cervical anastomosis (11). Previous researches reported the risk of anastomotic leakage at the end of the TST and TS anastomoses (9). Other studies found that TST and TS anastomoses with a linear stapler had low rates of stricture and anastomotic leakage (12).

CS, TST and TS anastomoses have advantages and disadvantages in clinical practice, but few have compared them in a cervical anastomotic operation. In this study, we systematically analyzed and compared the clinical application of these three methods in minimally invasive resection of esophageal cancer. We present the following article in accordance with the STROBE reporting checklist (available at http://dx.doi.org/10.21037/atm-20-7278).

\section{Methods}

\section{Patients}

Patients diagnosed as having esophageal cancer at Qilu Hospital of Shandong University between April 2010 and June 2012 were recruited using the following inclusion criteria: (I) diagnosed as esophageal cancer, and underwent minimally invasive esophagectomy (MIE) without preoperative radiotherapy and chemotherapy, using standard chest and abdominal procedures of thoracoscopy and laparoscopy, anastomosis of tube stomach and esophagus in the neck; (II) no other severe disease that could affect prognosis; diseases that may affect prognosis include: (i) diabetic patients with organ damage (such as diabetic nephropathy), (ii) preoperative color photos or CT of the abdomen suggest liver cirrhosis with lower albumin; (iii) severe cardiopulmonary dysfunction before surgery cannot tolerate surgery (FEV1 $<50 \%, \mathrm{EF}<50 \%$ ). (III) Complete resection (R0 resection), and pathological stage $\leq$ IIIA according to the 7 th edition of the American Joint Committee on Cancer Tumor-Node-Metastasis (TNM) staging system; (IV) 2-field or 3-field lymph node dissection; and (V) severe comorbidities. This retrospective study was approved by the Institutional Review Board of Qilu Hospital of Shandong University (No. KYLL-2019-
186) and all patients gave written informed consent. We confirm that this study conformed to the provisions of the Declaration of Helsinki (as revised in 2013)

\section{Surgical procedures}

All operations were performed by the same team, consisting of the same surgeon and two skilled assistant surgeons.

TST was performed as described previously (13). Briefly, we placed 3 or 4 suspension sutures in the posterior wall of the remnant esophagus and the gastric tube. Next, we pulled up the anastomosis, clamped the suspended esophagus and stomach with a linear cutting suture device, and cut and sutured. We then placed three sutures along the edge of the first cut on the right side, pulled up the sutures to clamp the esophagus and tubular stomach with a straight-line cutting suture device, and cut and closed them. Finally, we cut and closed the left side of the esophagus and stomach, and completed the circumferential closure of the whole triangular anastomosis, embedding the suture at the join of each cutting and suturing.

TS was performed as follows. First, we placed the deepest point on the top of the stapler in the esophagus and the tube stomach, and then sutured. Next, we raised the gastric tube to the left neck incision, and created a $1-\mathrm{cm}$ gastrostomy at the posterior wall of the gastric tube. We placed the anvil of the stapler in the remnant esophagus, and the staple cartridge in the gastric tube. Apply the second firing of the stapler transversely.

CS was performed as follows. Clamp the broken end of esophagus with a bag clamp, and suture the broken end of esophagus with a bag line. The stapler thimble was placed at the cut end of the esophagus, and the stapler thimble was fixed by a purse string suture. Create a $2-\mathrm{cm}$ incision at the top of the tube stomach. When the stapler is inserted the tube stomach, select the proper position of the great curvature side of the stomach and perform mechanical anastomosis with the stapler thimble. Cut and close the incision on the top of the stomach.

\section{Follow-up}

Patients who were discharged successfully were first followed up in the month after the operation. In the first year after the operation, follow-up occurred every 3 months. In the second and third years, follow-up was reduced to every 6 months. In the fourth to fifth years, follow-up was annual. At the first follow-up, upper gastrointestinal 
contrast examination and routine hematology and blood biochemistry were carried out; at other postoperative follow-up, chest and abdomen enhanced computed tomography (CT) and hematology examinations were carried out.

\section{Definition of postoperative complications}

Anastomotic leakage was defined as the presence of extraluminal contrast by CT and swallow test, dehiscence or fistulae by endoscopy, or loss of saliva. The definition of postoperative gastroesophageal reflux referred to the feeling of the patient: no reflux symptoms, mild reflux, moderate reflux and severe reflux. The definition of postoperative anastomotic stenosis mainly referred to the eating experience of the patient: no eating obstruction or an eating obstruction. Other postoperative complications, including pleural effusion, chylothorax, pulmonary infection and hoarseness, were determined according to clinical diagnostic criteria.

\section{Management of anastomotic leakage}

After the operation, if anastomotic leakage occurs, the neck incision must be first removed, and then fully drained. Next, chest CT should be performed to evaluate whether the leakage has entered the thoracic cavity and if required, adequate drainage of the chest and mediastinum should be performed. Patients with postoperative anastomotic leakage also received gastrointestinal decompression and adequate nutritional support.

\section{Statistical analysis}

Statistical analysis was performed with SPSS 19.0 software (Chicago, IL, USA) with $\mathrm{P}<0.05$ indicating significance. The Chi-square test was used to compare the TST, TS and CS groups. Kaplan-Meier method and log-rank test were used to compare the survival of the three groups. Multivariate Cox regression analysis was used to identify prognostic factors.

\section{Results}

A total of 224 patients were reviewed, comprising 127 males (56.7\%) and 97 females (43.3\%); 87 (38.8\%) patients underwent CS, 78 (34.8\%) underwent TS and 59 (26.4\%) underwent TST (Figure 1). The screening and enrollment of patients are shown in Figure 2.Their characteristics are summarized in Table 1. There were no significant differences among the three groups, except for tumor pathological stage and tumor location. The location and pathological stage of the tumor affected the choice of operation (Figure 3).

The anastomotic time for the CS,TS and TST anastomotic techniques was 24.45 (23.64-25.25) $\mathrm{min}, 15.82$ (15.10-16.54) min and 21.39 (20.46-22.32) min, respectively; the operation time was $198.82(195.66-210.97) \mathrm{min}, 187.00$ (183.42-190.58) $\mathrm{min}$ and $185.41(180.81-190.00) \mathrm{min}$, respectively; hospitalization was $18.63(16.47-20.79)$ days, 13.89 (12.71-15.05) days and 16.09 (14.50-17.67) days, respectively. There were significant differences in these time periods among the groups (all $\mathrm{P}<0.001$ ), but there was no difference among them in the albumin content at 1 month after operation $(\mathrm{P}=0.226)$ (Table 2, Figure 4).

As shown in Table 3, the incidence of anastomotic leakage was $12.6 \%(11 / 87)$ in the CS group, 2.6\% (2/78) in the TS group, and $5.1 \%(3 / 59)$ in the TST group, with significant differences among the groups $(\mathrm{P}=0.033)$. The incidence of gastroesophageal reflux was $19.5 \%$ (moderate : $4.6 \%$; mild: $14.9 \%$ ) in the CS group, $44.9 \%$ (middle: 6.4\%; mild: $38.5 \%$ ) in the TS group, and $13.6 \%$ (middle: $1.7 \%$; mild: $11.9 \%$ ) in the TST group, with significant differences among the groups $(\mathrm{P}<0.001)$. The incidence of pleural effusion was $1.1 \%(1 / 87)$ in the CS group, $6.4 \%$ $(5 / 78)$ in the TS group and $0 \%(0 / 59)$ in the TST group, with significant differences among the groups $(\mathrm{P}=0.037)$. However, the rates of anastomotic stenosis (CS group: 18.4\%; TS group: $16.7 \%$; TST group:5.1\%), pulmonary infection (CS group: $8.0 \%$; TS group: $5.1 \%$; TST group: $6.8 \%$ ), chylothorax (CS group: $0 \%$; TS group: $2.6 \%$; TST group: $0 \%$ ) and hoarseness (CS group: $6.9 \%$; TS group: $5.1 \%$; TST group :3.4\%) were not different among the groups. No patients died during the follow-up.

Both anastomotic reinforcement and anastomotic suspension can be used in the three kinds of anastomotic methods. Surgeons decided to perform which technique independently. The relationship between operation and anastomotic complications is shown in Table 4. Among 16 patients with anastomotic leakage, 14 had undergone the operation of anastomotic suspension (Figure 5). Among 208 patients without anastomotic leakage, only 53 had undergone anastomotic suspension. Comparison of these two groups showed a significant difference $(\mathrm{P}<0.001)$. However, there was no significant differences in the operation of anastomotic suspension and postoperative 
A
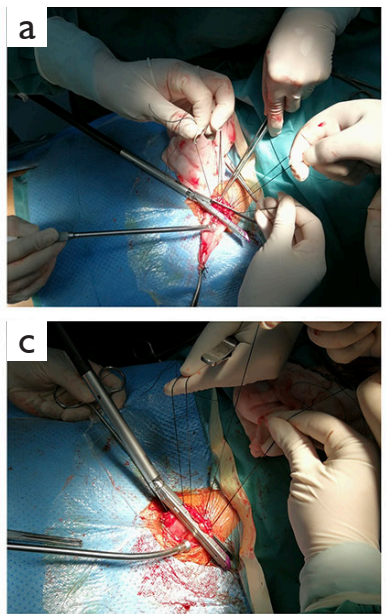
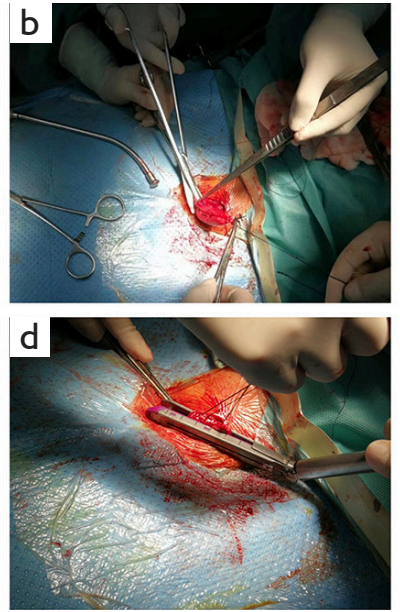

B
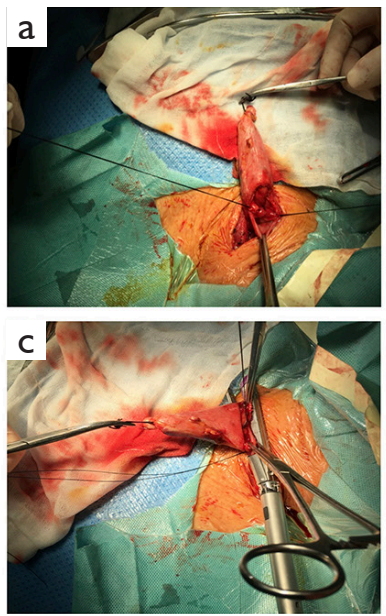
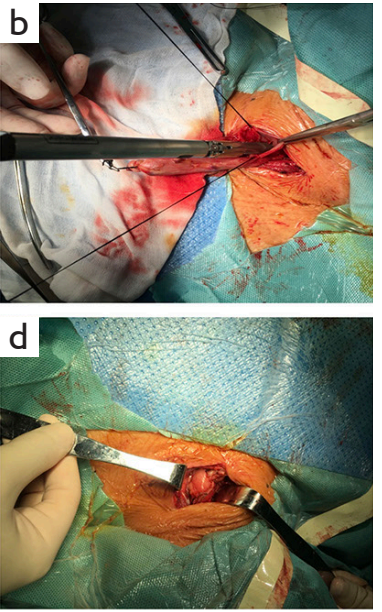

C
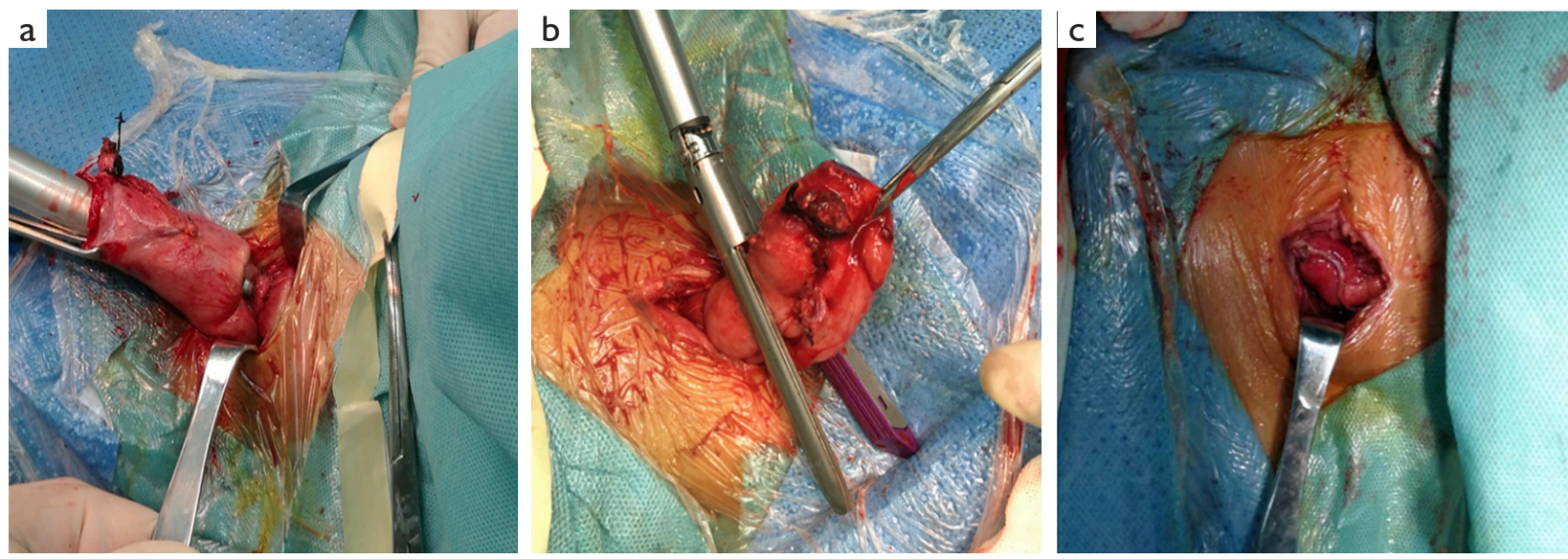

Figure 1 Schematic diagram of three kinds of anastomosis. (A) Triangulating stapling using a linear stapler. (B) T-shape stapling anastomosis using a linear stapler. (C) Circular anastomosis using a circular stapler.

complications of gastroesophageal reflux and anastomotic stenosis $(\mathrm{P}=0.478, \mathrm{P}=0.858$, respectively). Among 16 patients with anastomotic leakage, 5 had undergone the operation of anastomotic reinforcement, and among 208 patients without anastomotic leakage, 150 had undergone anastomotic reinforcement. Comparison of these two groups showed a significant difference $(\mathrm{P}=0.001)$. Similarly, there was a significant difference in the operation of anastomotic reinforcement and postoperative complications of gastroesophageal reflux and anastomotic stenosis $(\mathrm{P}<0.001, \mathrm{P}=0.045$, respectively).

We further compared the incidence of anastomotic leakage for the different surgical methods. The rate of anastomotic leakage was $45 \%$ in the patients with anastomosis suspension and $3 \%$ in the patients without it in the CS group $(\mathrm{P}<0.001), 9.5 \%$ versus $0 \%$ in the TS group $(\mathrm{P}=0.018)$, and $11.5 \%$ versus $0 \%$ in the TST group
$(\mathrm{P}=0.045)$. The rate of anastomotic leakage was $6.3 \%$ in the patients with anastomotic reinforcement and $29.2 \%$ in the patients without it in the $\mathrm{CS}$ group $(\mathrm{P}=0.04), 1.8 \%$ versus $4.5 \%$ in the TS group $(\mathrm{P}=0.488)$ and $0 \%$ versus $13.0 \%$ in the TST group ( $\mathrm{P}=0.026$, Table 5$)$.

We performed univariate and multivariate survival analyses in 224 patients. Of them, 84 (37.5\%) died within 5 years after operation, and tumor relapse occurred during follow-up in $88(39.3 \%)$ patients. Kaplan-Meier analyses compared with log-rank test were used to calculate the effect of the three types of cervical anastomosis on overall survival (OS) and disease-free survival (DFS). Univariate analysis demonstrated no difference in 5-year OS (CS group: $33.3 \%$; TS group: $35.9 \%$; TST group: $44.1 \%$, $\mathrm{P}=0.245$ ) or DFS (CS group: $35.6 \%$; TS group: $37.2 \%$; TST group: $45.6 \%, \mathrm{P}=0.282$ ) (Figure 6). Furthermore, multivariate analysis identified only TNM clinical 


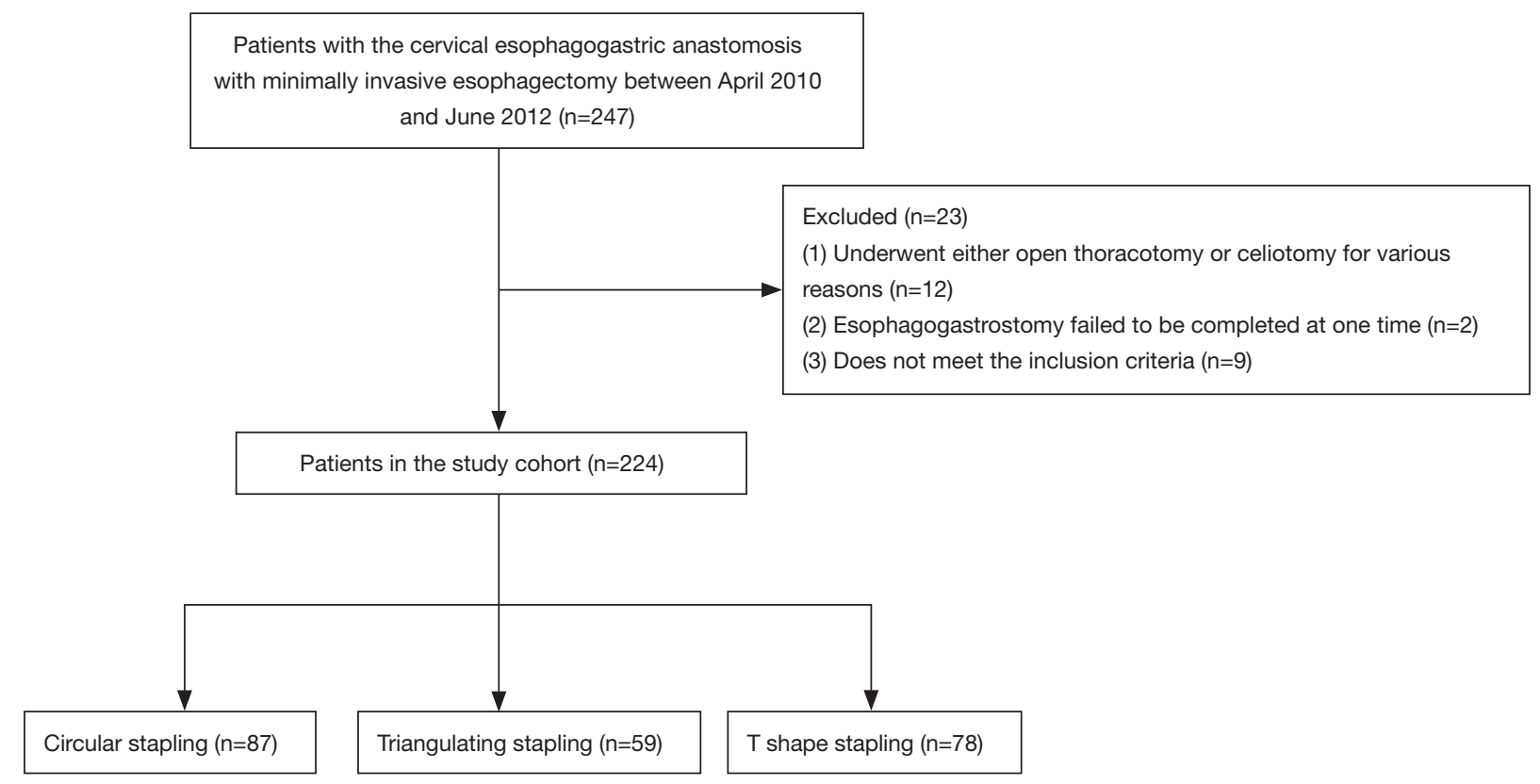

Figure 2 CONSORT flowchart of the patients enrolled in this study.

Table 1 Comparative study on the data of patients with three kinds of anastomoses

\begin{tabular}{|c|c|c|c|c|c|c|}
\hline Variable & Subgroup & Total & CS & TS & TST & $P$ value \\
\hline Sex & Female & 97 & 40 & 28 & 29 & \\
\hline \multirow[t]{2}{*}{ Age } & $<60 y$ & 126 & 43 & 49 & 34 & 0.216 \\
\hline & $\geq 60 y$ & 96 & 44 & 29 & 25 & \\
\hline Tumor location & Lower & 60 & 19 & 20 & 21 & \\
\hline \multirow[t]{3}{*}{ TNM staging } & 0 & 8 & 4 & 4 & 0 & 0.041 \\
\hline & I & 69 & 33 & 20 & 16 & \\
\hline & II & 100 & 38 & 39 & 23 & \\
\hline
\end{tabular}

TST, triangulating stapling; TS, T-shape stapling; CS, circular stapling technique.

stage $(\mathrm{P}<0.001)$ as an independent prognostic factor for progression-free survival.

\section{Discussion}

CS is one of the most widely used anastomotic methods because the stapling technique is simpler and easier (4). Hand-sewn anastomosis has also been used by many surgeons and achieves good results. A comparative study showed that the clinical effect of these two types of anastomosis was equivalent (14). Therefore, circular tubular anastomosis has become the standard method of cervical anastomosis. In this study, the anastomotic time, operative time and hospitalization time of the TS group and TST group were lower than those of the CS group, but there was no statistical difference in albumin content among the three 

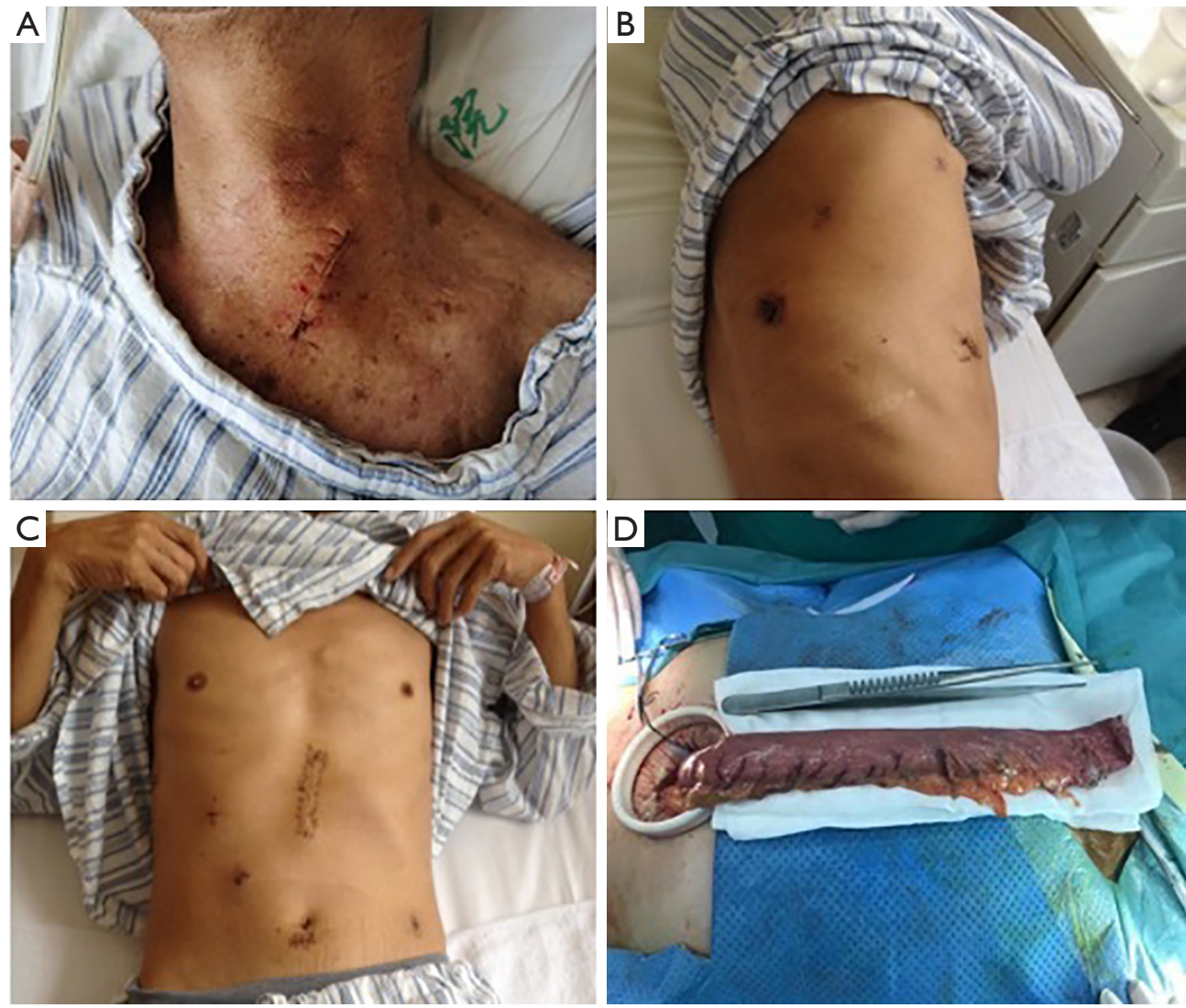

Figure 3 Sketch of surgical incision and picture of tubular stomach. (A) Neck incision in minimally invasive esophagectomy for cancer. (B) Chest incision. (C) Abdominal incision. (D) Photos of the tubular stomach.

Table 2 Comparative study of three kinds of anastomosis in operation

\begin{tabular}{lcccc}
\hline Variable & CS & TS & TST & P value \\
\hline Anastomotic time $(\mathrm{min})$ & $24.45(23.64-25.25)$ & $15.82(15.10-16.54)$ & $21.39(20.46-22.32)$ & 0.000 \\
Operation time $(\mathrm{min})$ & $198.82(195.66-210.97)$ & $187.00(183.42-190.58)$ & $185.41(180.81-190.00)$ & 0.000 \\
Hospital stay $(\mathrm{d})$ & $18.63(16.47-20.79)$ & $13.89(12.71-15.05)$ & $16.09(14.50-17.67)$ & 0.000 \\
Albumin content ${ }^{*}(\mathrm{~g} / \mathrm{L})$ & $41.09(40.36-41.82)$ & $40.72(39.95-41.50)$ & $40.09(39.22-40.97)$ & 0.226 \\
\hline
\end{tabular}

*: albumin content one month after operation. TST, triangulating stapling; TS, T-shape stapling; CS, circular stapling technique.

groups, indicating that the different methods had no effect on the overall postoperative nutritional recovery of the patients, perhaps due to a small-tube jejunostomy for each patient. The reason for the longer average hospitalization time in the CS group was the higher incidence of anastomotic leakage.

Despite recent progress in the development of surgical techniques, the rate of anastomotic leakage is still high. Anastomotic leakage can cause many complications, such as empyema and pleural effusion. For gastrointestinal anastomoses, the risk of cervical anastomotic leak is the highest (15). Our results showed that the rate of anastomotic leakage with the circular stapler was $7.1 \%$ in MIE. Because of the limited space in a neck operation, the choice of anastomotic technique is very important for postoperative anastomotic healing. TS and TST are two new methods of cervical anastomosis that are completely different from CS. In this study, for the first time we retrospectively compared 

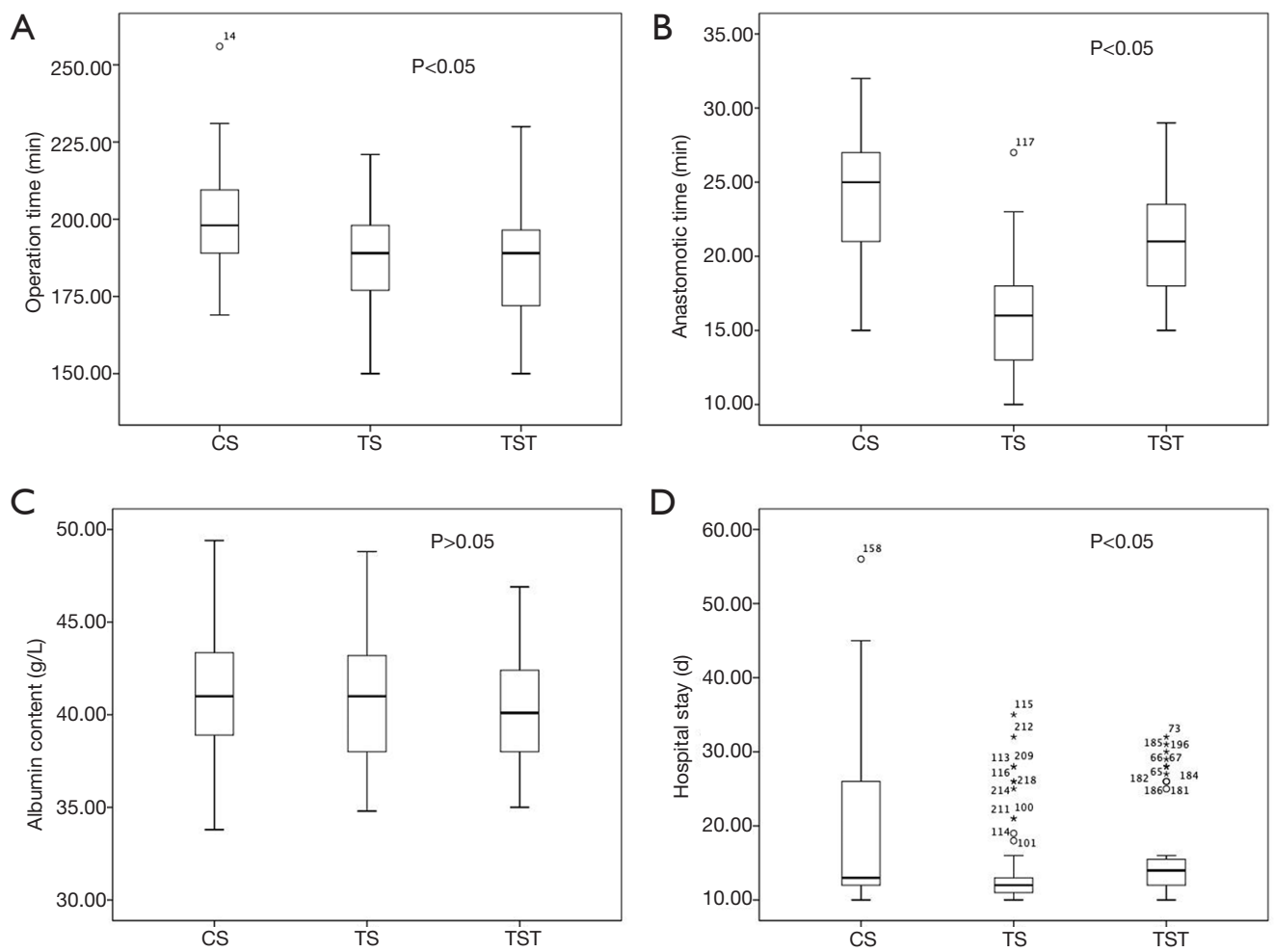

Figure 4 Comparison of clinical outcomes for the three types of anastomosis.

Table 3 Comparative study on postoperative complications of three anastomotic methods

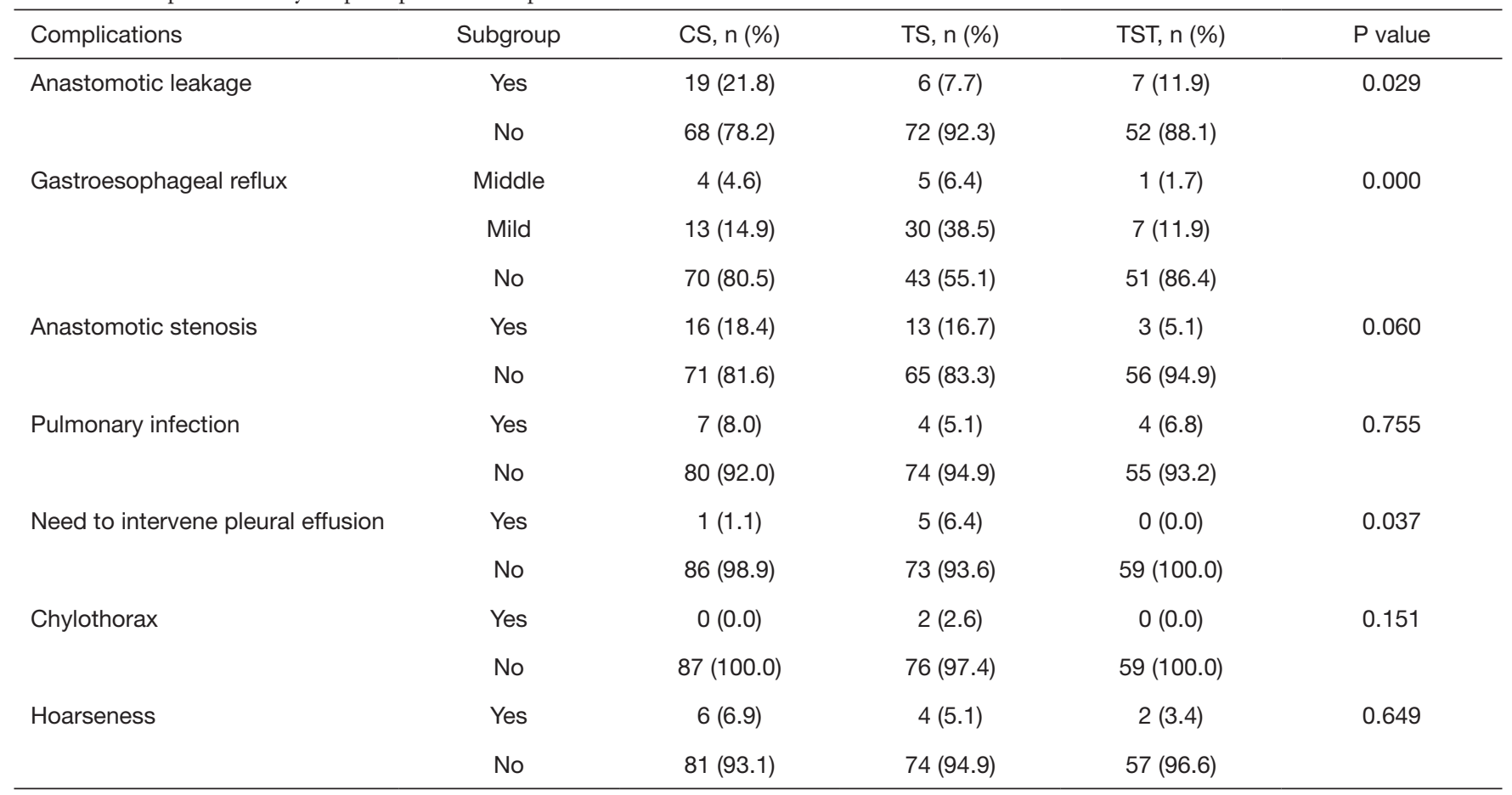

TST, triangulating stapling; TS, T-shape stapling; CS, circular stapling technique. 
Table 4 The relationship between operation and anastomotic complications

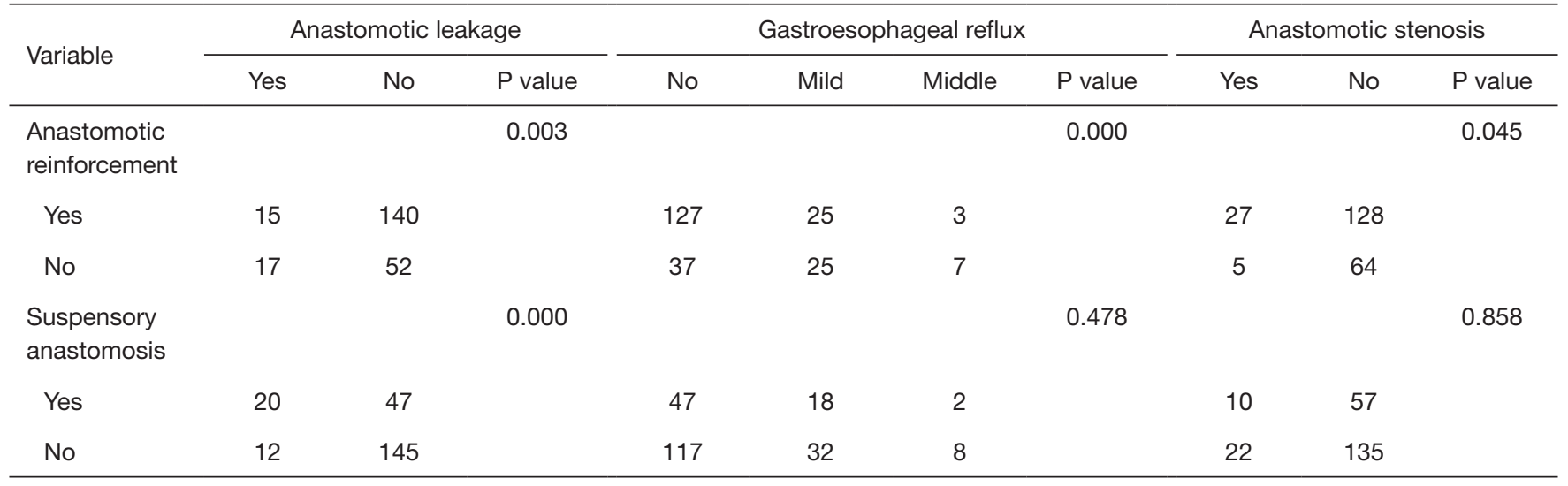
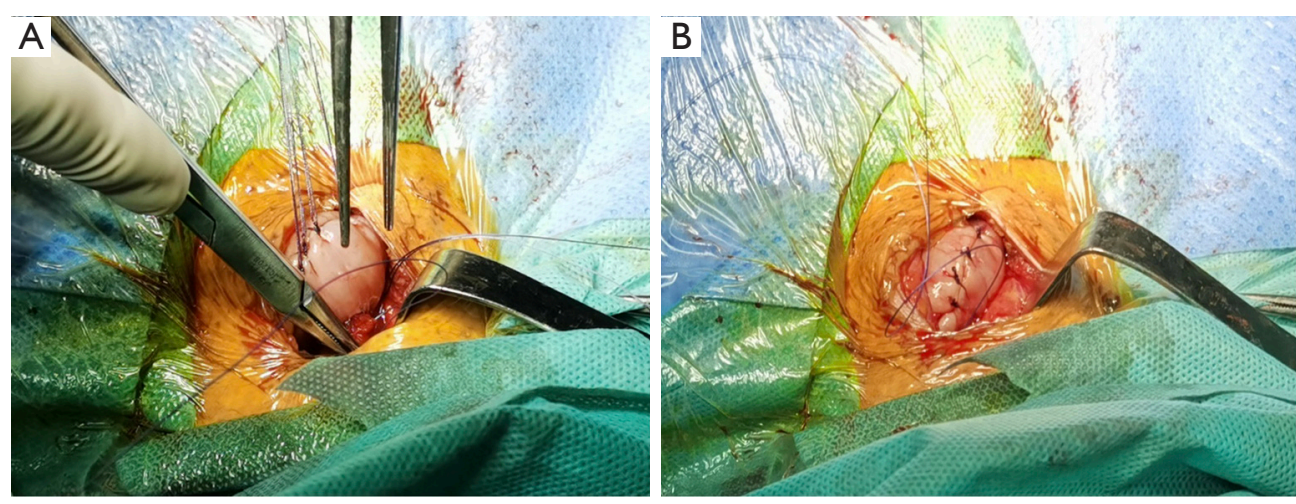

Figure 5 Operation sketch of anastomotic reinforcement and anastomotic suspension. (A) Anastomotic reinforcement. (B) Anastomotic suspension.

Table 5 Comparative study on the probability of anastomotic leakage in different measures of anastomotic treatment

\begin{tabular}{|c|c|c|c|c|c|c|}
\hline \multirow{3}{*}{ Operation } & \multicolumn{6}{|c|}{ Anastomotic leakage } \\
\hline & \multicolumn{2}{|c|}{ CS } & \multicolumn{2}{|c|}{ TS } & \multicolumn{2}{|c|}{ TST } \\
\hline & $\%$ & $P$ value & $\%$ & $P$ value & $\%$ & $P$ value \\
\hline $\begin{array}{l}\text { Suspensory } \\
\text { anastomosis }\end{array}$ & & 0.004 & & 0.022 & & 0.001 \\
\hline Yes & 45.0 & & 16.7 & & 26.9 & \\
\hline $\begin{array}{l}\text { Anastomotic } \\
\text { reinforcement }\end{array}$ & & 0.06 & & 0.217 & & 0.294 \\
\hline Yes & 14.3 & & 5.4 & & 8.3 & \\
\hline No & 42.7 & & 13.6 & & 17.4 & \\
\hline
\end{tabular}

TST, triangulating stapling; TS, T-shape stapling; CS, circular stapling technique. 

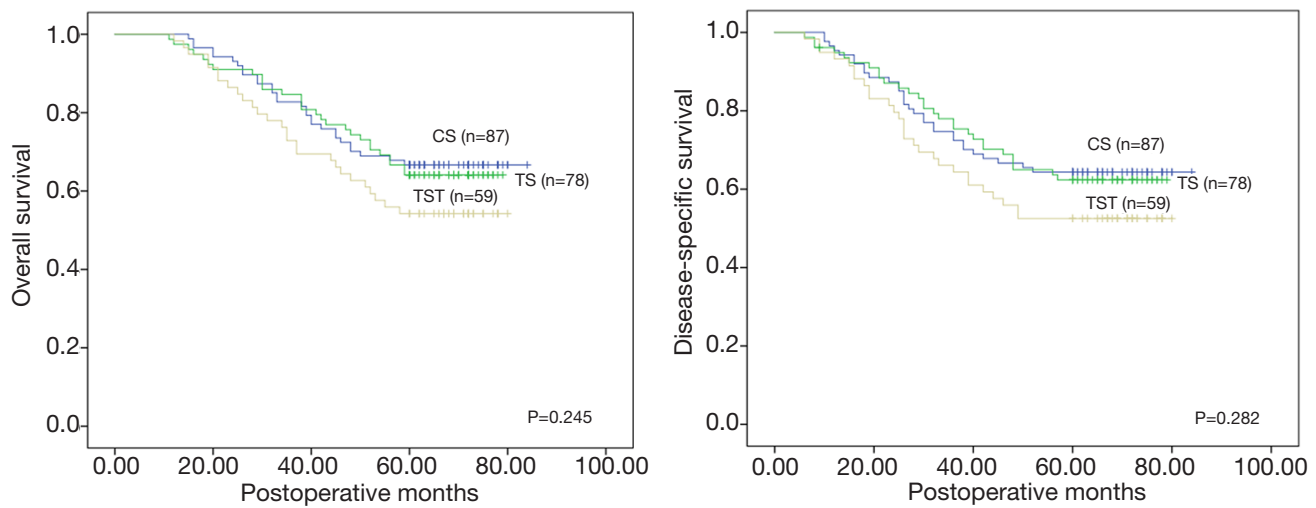

Figure 6 Comparison of the 5-year overall survival and disease-free survival for the three anastomotic techniques.

TS, TST and CS.

It is reported that the incidence of anastomotic leakage with TS and TST was better than that with CS (16). Yoshida et al. performed TST in 33 patients, and the rate of anastomotic leakage reduced to $1.0 \%$, compared with $13.3 \%$ after hand-sewn anastomosis (9). Huang et al. reported a decrease of the incidence anastomotic leakage in a TS group compared to a CS group, although without significant difference (17). Our results are consistent with these previous studies.

We further analyzed the cause of the high incidence of anastomotic leakage in the CS group. In addition to the different anastomotic methods, we found that anastomotic suspension and anastomotic reinforcement were closely related to the occurrence of anastomotic leakage. The rate of anastomotic leakage can be increased by anastomotic suspension, but there was no statistical relationship between anastomotic suspension and anastomotic reflux or stenosis. Strengthening the anastomosis can decrease the rate of anastomotic leakage and anastomotic reflux, but increase the rate of anastomotic stenosis. Our results showed that among the three anastomotic methods, anastomotic suspension was positively associated with anastomotic leakage. The operation of anastomotic reinforcement reduced the rate of anastomotic leakage in the CS and TST groups, and there was no significant correlation with anastomotic leakage in the TS group. Our study further showed that the rate of anastomotic leakage in the CS group was higher than that in TS and TST groups, which indicated that these techniques were better than CS in reducing anastomotic leakage in cervical anastomosis.

When anastomotic leakage occurs, some doctors use the operation of suspending the anastomoses to prevent leakage flowing into the mediastinum and forming mediastinal abscess. The proportion of pleural effusion was significantly higher in the TS group compared with the CS group. Among the six patients with pleural effusion that need to be treated, five underwent TS anastomosis and none of them were anastomotic suspension patients. We tested the blood supply at the anastomotic site, and found that it was adequate. Our results showed that the incidence of anastomotic leakage was higher in patients with anastomotic suspension, which may be due to the fact that suspension of the anastomotic site changed the straight-line state of the esophagus, making the digestive tract curved, and the location of the suspended anastomotic site was at the thyroid gland and neck muscle, narrowing the anastomotic space and leads to a restricted blood supply to the tube stomach. We recorded the location of anastomotic leakage in patients with a CS anastomotic pattern. The location of ischemic necrosis in $95 \%$ of patients with anastomotic leakage was in the suspended position, and all of them had residual gastric necrosis. In contrast, the diameter of the TS anastomotic orifice is larger $(\sim 5 \mathrm{~cm})$, the area of the anastomotic orifice is increased, vascular anastomotic branches of the tube and stomach are increased, and blood supply is significantly increased, leading to a reduced rate of anastomotic leakage.

The rate of anastomotic stenosis was not significantly different among the three anastomotic methods, but the rate of anastomotic stenosis in the TST group was lower than that in the other two groups, which may be related to the lesser damage of the mucosa caused by the triangular anastomotic pattern (18). Compared with the other two groups, the symptoms of anastomotic reflux were more severe in the TS group. In traditional end-to-side 
anastomosis, the diameter of the anastomotic orifice is close to the diameter of the cervical esophagus $(\sim 3 \mathrm{~cm})$. In the CS group, the shape of the anastomosis is round and the diameter of anastomosis is controllable. In the TS group, the shape of the anastomosis is linear, and in the TST group the shape is triangular; we believe that this leads to the differences in the incidence of postoperative reflux.

After follow-up of the 5-year OS and DFS rates of the three groups, we found no differences, indicating no differences in long-term surgical effects of the three types of anastomosis. Our data also showed no statistical differences in tumor location and tumor stage among the three groups. There was no difference in 30-90-day mortality. Even if the patients had anastomotic leakage, after active drainage and nutrition, they could recover and be discharged from hospital. Although anastomotic suspension increased the probability of anastomotic leakage, it prevented the contents of the anastomotic leakage from entering the chest and reduced the risk of death.

In summary, cervical esophagogastric anastomosis using the TST and TS techniques can be considered as safe and feasible, and improve short-term outcomes with low rates of anastomotic leakage and stenosis despite an increased rate of anastomotic reflux.

\section{Acknowledgments}

Funding: None.

\section{Footnote}

Reporting Checklist: The authors have completed the STROBE reporting checklist. Available at http://dx.doi. org/10.21037/atm-20-7278

Data Sharing Statement: Available at http://dx.doi. org/10.21037/atm-20-7278

Conflicts of Interest: All authors have completed the ICMJE uniform disclosure form (available at http://dx.doi. org/10.21037/atm-20-7278). The authors have no conflicts of interest to declare.

Ethical Statement: The authors are accountable for all aspects of the work in ensuring that questions related to the accuracy or integrity of any part of the work are appropriately investigated and resolved.The study protocol was approved by Ethics Boards of Qilu Hospital (No.
KYLL-2019-186), and tissue specimen acquisition was carried out in accordance with the institutional guidelines. All subjects signed written informed consent. We confirm that this study conformed to the provisions of the Declaration of Helsinki (as revised in 2013).

Open Access Statement: This is an Open Access article distributed in accordance with the Creative Commons Attribution-NonCommercial-NoDerivs 4.0 International License (CC BY-NC-ND 4.0), which permits the noncommercial replication and distribution of the article with the strict proviso that no changes or edits are made and the original work is properly cited (including links to both the formal publication through the relevant DOI and the license). See: https://creativecommons.org/licenses/by-nc-nd/4.0/.

\section{References}

1. van der Horst S, de Maat MFG, van der Sluis PC, et al. Extended thoracic lymph node dissection in roboticassisted minimal invasive esophagectomy (RAMIE) for patients with superior mediastinal lymph node metastasis. Ann Cardiothorac Surg. 2019;8:218-25.

2. Ercan S, Rice TW, Murthy SC, et al. Does esophagogastric anastomotic technique influence the outcome of patients with esophageal cancer? J Thorac Cardiovasc Surg 2005;129:623-31.

3. Alanezi K, Urschel JD. Mortality secondary to esophageal anastomotic leak. Ann Thorac Cardiovasc Surg 2004;10:71-5.

4. Lee Y, Fujita H, Yamana H, et al. Factors affecting leakage following esophageal anastomosis. Surg Today 1994;24:24-9.

5. Xu QR, Wang KN, Wang WP, et al. Linear stapled esophagogastrostomy is more effective than handsewn or circular stapler in prevention of anastomotic stricture: a comparative clinical study. J Gastrointest Surg 2011;15:915-21.

6. Santos RS, Raftopoulos Y, Singh D, et al. Utility of total mechanical stapled cervical esophagogastric anastomosis after esophagectomy: a comparison to conventional anastomotic techniques. Surgery 2004;136:917-25.

7. Wong J, Cheung H, Lui R, et al. Esophagogastric anastomosis performed with a stapler: the occurrence of leakage and stricture. Surgery 1987;101:408-15.

8. Orringer MB, Marshall B, Iannettoni MD. Eliminating the cervical esophagogastric anastomotic leak with a sideto-side stapled anastomosis. J Thorac Cardiovasc Surg 
2000;119:277-88.

9. Yoshida N, Baba Y, Watanabe M, et al. Triangulating stapling technique covered with the pedicled omental flap for esophagogastric anastomosis: a safe anastomosis with fewer complications. J Am Coll Surg 2015;220:e13-6.

10. Nakata K, Nagai E, Ohuchida K, et al. Outcomes of cervical end-to-side triangulating esophagogastric anastomosis with minimally invasive esophagectomy. World J Surg 2015;39:1099-104.

11. Dong YN, Zhang L, Sun N, et al. Novel T-shaped linearstapled intrathoracic esophagogastric anastomosis for minimally invasive Ivor Lewis esophagectomy. Ann Thorac Surg 2015;99:1459-63.

12. Noshiro H, Urata M, Ikeda O, et al. Triangulating stapling technique for esophagogastrostomy after minimally invasive esophagectomy. Surgery 2013;154:604-10.

13. Hayata K, Nakamori M, Nakamura M, et al. Circular stapling versus triangulating stapling for the cervical esophagogastric anastomosis after esophagectomy in patients with thoracic esophageal cancer: A prospective,

Cite this article as: Tian Y, Li L, Li S, Tian H, Lu M. Comparison of circular stapling, triangulating stapling and T-shape stapling for cervical anastomosis with minimally invasive esophagectomy. Ann Transl Med 2020;8(24):1679. doi: 10.21037/atm-20-7278 randomized, controlled trial. Surgery 2017;162:131-8.

14. Beitler AL, Urschel JD. Comparison of stapled and hand-sewn esophagogastric anastomoses. Am J Surg 1998;175:337-40.

15. Korst RJ, Port JL, Lee PC, et al. Intrathoracic manifestations of cervical anastomotic leaks after transthoracic esophagectomy for carcinoma. Ann Thorac Surg 2005;80:1185-90.

16. Li J, Shen Y, Tan L, et al. Cervical triangulating stapled anastomosis: technique and initial experience. J Thorac Dis 2014;6 Suppl 3:S350-4.

17. Huang C, Xu X, Zhuang B, et al. A comparison of cervical delta-shaped anastomosis and circular stapled anastomosis after esophagectomy. World J Surg Oncol 2017;15:31.

18. Furukawa Y, Hanyu N, Hirai K, et al. Usefulness of automatic triangular anastomosis for esophageal cancer surgery using a linear stapler (TA-30). Ann Thorac Cardiovasc Surg 2005;11:80-6.

(English Language Editor: K. Brown) 